\title{
EVALUATION OF IMPACT OF COMBINED ORTHODONTIC AND ORTHOGNATHIC TREATMENT PROCEDURES ON THE LIFE QUALITY OF PATIENTS WITH SKELETAL MALOCCLUSION
}

\section{Dr Ranjith Raveendran*}

Professor, Department of Orthodontics, Government Dental College, Pariyaram, Kannur, Kerala, India. *Corresponding Author

Oral and Maxillofacial Surgeon, Government Hospital, Thalassery, Kannur,Kerala, India.

Associate Professor, Department of Periodontics, KMCT Dental College, Manassery, Kozhikode, Kerala, India.

ABSTRACT Background: The number of patients undergoing combined orthodontic treatment and orthognathic surgery is increasing. Hence, this study aimed to examine the impact of combined orthodontic and orthognathic treatment procedures on the life quality of patients with skeletal malocclusion.

Materials and methods: 15 patients who had to undergo combined orthodontic and orthognathic treatment procedures were subjected to 22-item Orthognathic Quality of Life Questionnaire customised for the study. The questions were grouped into 4 domains - aesthethic, awareness, pschyological and social domains. They were assessed in three time lines - first when they are explained and made aware of the treatment plan (T0), Tl when the orthodontic treatment is almost complete, and the patient is made ready for orthognathic surgery, then 6 months after the completion of the combined orthodontic-orthognathic surgery (T2).

Results: Out of 15 patients, 9 were females and 6 were males between the age of 18-25 years. As the treatment progressed changes were noted in the patient's attitude. During Tl significant change was observed in functional outcome - chewing. At T2 significant changes were observe in esthetic, functional, social and psychological components $(p<0.001)$. The treatment outcome did create a positive change in the patients by correcting their skeletal and dental abnormalities.

Conclusion: The esthetic, functional, social and psychological outcomes of patients who have undergone combined orthodontic and orthognathic treatments are better post operatively.

KEYWORDS : Life quality; orthodontic; orthognathic; combined; questionnare

\section{INTRODUCTION}

To achieve a high level of satisfaction among our patients seeking treatments it is prudent to communicate effectively and create awareness in the patients prior to any treatment procedure under your guidance and care. Bailey et al. explains that including patients in the decision-making process increases their awareness and acceptance of the final results. ${ }^{1}$ Several studies have shown that educating the patients prior to treatment is needed to achieve a high level of satisfaction among those patients who dental treatments. ${ }^{1-6} \mathrm{~A}$ motivated patient will be willing to undergo the complete treatment suggested by the professional, if he is confident that the outcome will definitely improve the quality of his life.

The number of patients undergoing combined orthodontic treatment and orthognathic surgery is increasing. This may be partly due to the increased awareness created by the dental professional , the internet, and partly due to access to better treatment at affordable rates. The patient satisfaction and the change in quality of life after a combined orthodontic and orthognathic treatment for correction of skeletal malocclusion has not been evaluated in our centre before. So, we planned for an objective evaluation of patient prior to the treatment, during mid treatment and at the end of the combined treatment. Hence, this study aimed to examine the impact of combined orthodontic and orthognathic treatment procedures on the life quality of patients with skeletal malocclusion.

\section{Materials \& Methods}

The subjects underwent fixed appliance therapy from the Department of Orthodontics, Government Dental College, Pariyaram. They were subjected to orthognathic surgery from the Government Hospital, Thalassery, Kannur. The research protocol was described to patients and a written consent was obtained before participating in the study. This study has been conducted in full accordance with the World Medical Association Declaration of Helsinki.
Study Participants

A total of 15 patients were included in the study based on the inclusion and exclusion criteria. The exclusion criteria was the presence of a congenital deformity, history of facial trauma or removal of an appliance $<6$ months previously, patients who were scheduled to undergo orthognathic surgery without orthodontic treatment or with additional features, e.g., genioplasty or distraction device, and patients reluctant to participate in the study.

\section{Methodology}

Data were collected using self-administered 22-item Orthognathic Quality of Life Questionnaire(OQLQ a condition specific assessment)based on the study conducted by Faezeh Eslamipour et al in 2017. ${ }^{5}$ The Orthognathic Quality of Life Questionnaire (OQLQ) was developed as an instrument to estimate quality of life in patients treated with orthognathic surgery(Figure 1).It was developed in 2000 and validated in 2002 by Cunningham et al. ${ }^{7,8}$

OQLQ questionnaire consists of 22 questions rated on a 5point Likert scale ranging from "does not bother me at all"(score 0)to "bothers me a lot"(score 4). The total score is 0 to 88. A lower score indicates a better QOL and viceversa. Also, this questionnaire consists of 4 subscales:aesthetic impact (items $1,7,10,11$, and 14 , range 0 to 20 ), oral function(2 to 6 , range 0 to 20$)$, awareness \& psychological impact $(8,9,12$, and 13 ,range 0 to 16 ), and social impact(items 15 to 22 , range 0 to32).

We have modified the original OQLQ questionnaire to suit the population based on the social settings prevailing in this part of the country (Kerala State, India). The 22 questions were therefore divided into 4 domains: aesthetic impact oral function, Awareness \& psychological impact and social impact. A lower score indicates a better QOL and viceversa.

The questionnaire was distributed to the patients and asked 
them to complete it first during their routine visits in orthodontist office, when they are first explained and made aware of the treatment plan(T0),Tlis the time when the orthodontic treatment is almost complete, and the patient is made ready for orthognathic surgery, then 6 months after the complete orthodontic- orthognathic surgery is executed and the patient reports for review(T2).

\section{Statistical Methods}

All statistical procedures were performed using Statistical Package for Social Sciences (SPSS) 20.0.Calculations for power ( $80 \%$ ) of study was performed before commencement of the study. All quantitative variables expressed in mean and standard Deviation. Qualitative variables expressed as percentage. Shapiro- Wilk test was used for testing the normality assumption of the data. Paired $t$ test was done to find the association between variables. Probablility value $(p<0.05)$ will be considered statistically significant.

\section{RESULTS}

A total of 15 patients with dental-skeletal discrepancies participated in the study.Out of them 9 were females and 6 were males. They were between the age of $18-25$ years. Tables 1,2 and 3 shows the response to the questionnaire during the timelines T0, T1 and T2 respectively. Since the 22 questions were considered under 4 domains, comparisons between each timeline was done based on those categories.

After orthodontic treatment esthetic, social and psychological components showed no significant changes where as significant change was observed in functional outcome chewing. After surgical phase significant changes were observe in esthetic, functional, social and psychological components $(p<0.001)$.

Table 1: Patient response before the start of the treatment (T0)

\begin{tabular}{|c|c|c|c|c|c|c|c|c|}
\hline \multirow{2}{*}{$\begin{array}{l}\text { Sl } \\
\text { No. }\end{array}$} & \multirow[t]{2}{*}{ OQLQ } & \multirow{2}{*}{$\begin{array}{l}\mathbf{N} \\
\%\end{array}$} & \multicolumn{5}{|c|}{ Scores } & \multirow[t]{2}{*}{ Total } \\
\hline & & & 0 & 1 & 2 & 3 & 4 & \\
\hline \multirow[t]{2}{*}{1.} & \multirow{2}{*}{$\begin{array}{l}\text { I am self-conscious about the appearance of } \\
\text { my teeth }\end{array}$} & $\mathrm{n}$ & l & 2 & 3 & 5 & 4 & 15 \\
\hline & & $\%$ & $6.7 \%$ & $13.3 \%$ & $20.0 \%$ & $33.3 \%$ & $26.7 \%$ & $100.0 \%$ \\
\hline \multirow[t]{2}{*}{2.} & \multirow{2}{*}{$\begin{array}{l}\begin{array}{l}\text { I don't like seeing a side view of my face } \\
\text { (profile) }\end{array} \\
\end{array}$} & $\mathrm{n}$ & 1 & 0 & 3 & 6 & 5 & 15 \\
\hline & & $\%$ & $6.7 \%$ & $0.0 \%$ & $20.0 \%$ & $40.0 \%$ & $33.3 \%$ & $100.0 \%$ \\
\hline \multirow[t]{2}{*}{3.} & \multirow{2}{*}{$\begin{array}{l}\text { I spend a lot of time studying my face in the } \\
\text { mirror }\end{array}$} & $\mathrm{n}$ & l & 0 & 6 & 4 & 4 & 15 \\
\hline & & $\%$ & $6.7 \%$ & $0.0 \%$ & $40.0 \%$ & $26.7 \%$ & $26.7 \%$ & $100.0 \%$ \\
\hline \multirow[t]{2}{*}{4.} & \multirow{2}{*}{$\begin{array}{l}\text { I spend a lot of time studying my teeth in the } \\
\text { mirror }\end{array}$} & $\mathrm{n}$ & l & l & 5 & 4 & 4 & 15 \\
\hline & & $\%$ & $6.7 \%$ & $6.7 \%$ & $33.3 \%$ & $26.7 \%$ & $26.7 \%$ & $100.0 \%$ \\
\hline \multirow[t]{2}{*}{5.} & \multirow[t]{2}{*}{ I dislike having my photograph taken } & $\mathrm{n}$ & l & 2 & 4 & 5 & 3 & 15 \\
\hline & & $\%$ & $6.7 \%$ & $13.3 \%$ & $26.7 \%$ & $33.3 \%$ & $20.0 \%$ & $100.0 \%$ \\
\hline \multirow[t]{2}{*}{6.} & \multirow[t]{2}{*}{ I dislike being seen on video } & $\mathrm{n}$ & 2 & l & 5 & 5 & 2 & 15 \\
\hline & & $\%$ & $13.3 \%$ & $6.7 \%$ & $33.3 \%$ & $33.3 \%$ & $13.3 \%$ & $100.0 \%$ \\
\hline \multirow[t]{2}{*}{7.} & \multirow{2}{*}{$\begin{array}{l}\text { I am self conscious about my facial } \\
\text { appearance }\end{array}$} & $\mathrm{n}$ & l & l & 4 & 7 & 2 & 15 \\
\hline & & $\%$ & $6.7 \%$ & $6.7 \%$ & $26.7 \%$ & $46.7 \%$ & $13.3 \%$ & $100.0 \%$ \\
\hline \multirow[t]{2}{*}{8.} & \multirow[t]{2}{*}{ I do not like smiling when I am meeting people } & $\mathrm{n}$ & l & 0 & 7 & 6 & l & 15 \\
\hline & & $\%$ & $6.7 \%$ & $0.0 \%$ & $46.7 \%$ & $40.0 \%$ & $6.7 \%$ & $100.0 \%$ \\
\hline \multirow[t]{2}{*}{9.} & I have problems biting & $\mathrm{n}$ & 2 & 3 & 7 & 2 & l & 15 \\
\hline & & $\%$ & $13.3 \%$ & $20.0 \%$ & $46.7 \%$ & $13.3 \%$ & $6.7 \%$ & $100.0 \%$ \\
\hline 10. & I have problems chewing & $\mathrm{n}$ & 2 & 2 & 5 & 4 & 2 & 15 \\
\hline & & $\%$ & $13.3 \%$ & $13.3 \%$ & $33.3 \%$ & $26.7 \%$ & $13.3 \%$ & $100.0 \%$ \\
\hline 11. & There are some foods I avoid eating because & $\mathrm{n}$ & 4 & 3 & 7 & 1 & 0 & 15 \\
\hline & the way my teeth meet make them difficult & $\%$ & $26.7 \%$ & $20.0 \%$ & $46.7 \%$ & $6.7 \%$ & $0.0 \%$ & $100.0 \%$ \\
\hline 12. & I get pain in my face or jaw & $\mathrm{n}$ & 5 & 6 & 3 & 0 & l & 15 \\
\hline & & $\%$ & $33.3 \%$ & $40.0 \%$ & $20.0 \%$ & $0.0 \%$ & $6.7 \%$ & $100.0 \%$ \\
\hline 13. & I don't like eating in public places & $\mathrm{n}$ & 2 & 2 & 10 & l & 0 & 15 \\
\hline & & $\%$ & $13.3 \%$ & $13.3 \%$ & $66.7 \%$ & $6.7 \%$ & $0.0 \%$ & $100.0 \%$ \\
\hline 14. & I try to cover my mouth when I meet people for & $\mathrm{n}$ & 3 & 4 & 7 & 0 & l & 15 \\
\hline & the first time & $\%$ & $20.0 \%$ & $26.7 \%$ & $46.7 \%$ & $0.0 \%$ & $6.7 \%$ & $100.0 \%$ \\
\hline 15. & I worry about meeting people for the first time & $\mathrm{n}$ & 2 & 8 & 4 & 0 & 1 & 15 \\
\hline & & $\%$ & $13.3 \%$ & $53.3 \%$ & $26.7 \%$ & $0.0 \%$ & $6.7 \%$ & $100.0 \%$ \\
\hline 16. & I lack confidence when I am out socially & $\mathrm{n}$ & 2 & 0 & 7 & 5 & 1 & 15 \\
\hline & & $\%$ & $13.3 \%$ & $0.0 \%$ & $46.7 \%$ & $33.3 \%$ & $6.7 \%$ & $100.0 \%$ \\
\hline 17. & I often stare at other people's teeth & $\mathrm{n}$ & 2 & 6 & 5 & 2 & 0 & 15 \\
\hline & & $\%$ & $13.3 \%$ & $40.0 \%$ & $33.3 \%$ & $13.3 \%$ & $0.0 \%$ & $100.0 \%$ \\
\hline 18. & I often stare at other people's face & $\mathrm{n}$ & 2 & 3 & 6 & 3 & l & 15 \\
\hline & & $\%$ & $13.3 \%$ & $20.0 \%$ & $40.0 \%$ & $20.0 \%$ & $6.7 \%$ & $100.0 \%$ \\
\hline 19. & I worry that people will make hurtful comments & $\mathrm{n}$ & 2 & 5 & 7 & 0 & 1 & 15 \\
\hline & ppearance & $\%$ & $13.3 \%$ & $33.3 \%$ & $46.7 \%$ & $0.0 \%$ & $6.7 \%$ & $100.0 \%$ \\
\hline 20. & I sometimes get depressed about my & $\mathrm{n}$ & 2 & 2 & 7 & 4 & 0 & 15 \\
\hline & & $\%$ & $13.3 \%$ & $13.3 \%$ & $46.7 \%$ & $26.7 \%$ & $0.0 \%$ & $100.0 \%$ \\
\hline 21. & I sometimes think that people & $\mathrm{n}$ & 2 & l & 6 & 5 & 1 & 15 \\
\hline & are $:$ & $\%$ & $13.3 \%$ & $6.7 \%$ & $40.0 \%$ & $33.3 \%$ & $6.7 \%$ & $100.0 \%$ \\
\hline 22. & comments about my appearance really upset & $\mathrm{n}$ & 1 & l & 7 & 5 & l & 15 \\
\hline & me, although I know they are just joking & $\%$ & $6.7 \%$ & $6.7 \%$ & $46.7 \%$ & $33.3 \%$ & $6.7 \%$ & $100.0 \%$ \\
\hline
\end{tabular}


Questions 1-8 : esthetic component ; questions 9-12: functional component ; questions 13-16: social component ; questions 17-22: psychological component

Table 2: Patient response during the course of orthodontic therapy, but before the start of the orthognathic surgery (Tl)

\begin{tabular}{|c|c|c|c|c|c|c|c|c|}
\hline \multirow{2}{*}{$\begin{array}{l}\text { Sl } \\
\text { No. }\end{array}$} & \multirow[t]{2}{*}{ OQLQ } & \multirow{2}{*}{$\begin{array}{l}\mathbf{N} \\
\% \\
\end{array}$} & \multicolumn{5}{|c|}{ Scores } & \multirow[t]{2}{*}{ Total } \\
\hline & & & 0 & 1 & 2 & 3 & 4 & \\
\hline \multirow[t]{2}{*}{1.} & \multirow[t]{2}{*}{ I am self-conscious about the appearance of my teeth } & $\mathrm{n}$ & 0 & 1 & 3 & 5 & 6 & 15 \\
\hline & & $\%$ & $0.0 \%$ & $6.7 \%$ & $20.0 \%$ & $33.3 \%$ & $40.0 \%$ & $100.0 \%$ \\
\hline \multirow[t]{2}{*}{2.} & \multirow{2}{*}{ I don't like seeing a side view of my face (profile) } & $\mathrm{n}$ & 1 & 0 & 3 & 6 & 5 & 15 \\
\hline & & $\%$ & $6.7 \%$ & $0.0 \%$ & $20.0 \%$ & $40.0 \%$ & $33.3 \%$ & $100.0 \%$ \\
\hline \multirow[t]{2}{*}{3.} & \multirow[t]{2}{*}{ I spend a lot of time studying my face in the mirror } & $\mathrm{n}$ & 1 & 0 & 5 & 4 & 5 & 15 \\
\hline & & $\%$ & $6.7 \%$ & $0.0 \%$ & $33.3 \%$ & $26.7 \%$ & $33.3 \%$ & $100.0 \%$ \\
\hline \multirow[t]{2}{*}{4.} & \multirow[t]{2}{*}{ I spend a lot of time studying my teeth in the mirror } & $\mathrm{n}$ & 1 & 2 & 4 & 4 & 4 & 15 \\
\hline & & $\%$ & $6.7 \%$ & $13.3 \%$ & $26.7 \%$ & $26.7 \%$ & $26.7 \%$ & $100.0 \%$ \\
\hline \multirow[t]{2}{*}{5.} & \multirow[t]{2}{*}{ I dislike having my photograph taken } & $\mathrm{n}$ & 1 & 2 & 4 & 4 & 4 & 15 \\
\hline & & $\%$ & $6.7 \%$ & $13.3 \%$ & $26.7 \%$ & $26.7 \%$ & $26.7 \%$ & $100.0 \%$ \\
\hline \multirow[t]{2}{*}{6.} & \multirow[t]{2}{*}{ I dislike being seen on video } & $\mathrm{n}$ & 2 & 1 & 5 & 4 & 3 & 15 \\
\hline & & $\%$ & $13.3 \%$ & $6.7 \%$ & $33.3 \%$ & $26.7 \%$ & $20.0 \%$ & $100.0 \%$ \\
\hline \multirow[t]{2}{*}{7.} & \multirow{2}{*}{ I am self conscious about my facial appearance } & $\mathrm{n}$ & 1 & 0 & 6 & 4 & 4 & 15 \\
\hline & & $\%$ & $6.7 \%$ & $0.0 \%$ & $40.0 \%$ & $26.7 \%$ & $26.7 \%$ & $100.0 \%$ \\
\hline \multirow[t]{2}{*}{8.} & \multirow[t]{2}{*}{ I do not like smiling when I am meeting people } & $\mathrm{n}$ & l & 3 & 6 & 3 & 2 & 15 \\
\hline & & $\%$ & $6.7 \%$ & $20.0 \%$ & $40.0 \%$ & $20.0 \%$ & $13.3 \%$ & $100.0 \%$ \\
\hline \multirow[t]{2}{*}{9.} & I have problems biting & $\mathrm{n}$ & 4 & 7 & 1 & 2 & 1 & 15 \\
\hline & & $\%$ & $26.7 \%$ & $46.7 \%$ & $6.7 \%$ & $13.3 \%$ & $6.7 \%$ & $100.0 \%$ \\
\hline 10. & I have problems chewing & $\mathrm{n}$ & 2 & 9 & 3 & 0 & l & 15 \\
\hline & & $\%$ & $13.3 \%$ & $60.0 \%$ & $20.0 \%$ & $0.0 \%$ & $6.7 \%$ & $100.0 \%$ \\
\hline 11. & There are some foods I avoid ec & $\mathrm{n}$ & 9 & 5 & 0 & 0 & l & 15 \\
\hline & teeth meet make them difficult & $\%$ & $60.0 \%$ & $33.3 \%$ & $0.0 \%$ & $0.0 \%$ & $6.7 \%$ & $100.0 \%$ \\
\hline 12. & I get pain in my face or jaw & $\mathrm{n}$ & 2 & 5 & 6 & 1 & l & 15 \\
\hline & & $\%$ & $13.3 \%$ & $33.3 \%$ & $40.0 \%$ & $6.7 \%$ & $6.7 \%$ & $100.0 \%$ \\
\hline 13. & I don't like eating in public places & $\mathrm{n}$ & 2 & l & 4 & 4 & 4 & 15 \\
\hline & & $\%$ & $13.3 \%$ & $6.7 \%$ & $26.7 \%$ & $26.7 \%$ & $26.7 \%$ & $100.0 \%$ \\
\hline 14. & I try to cover my mouth when I meet people for the first & $\mathrm{n}$ & 3 & 4 & 6 & l & l & 15 \\
\hline & time & $\%$ & $20.0 \%$ & $26.7 \%$ & $40.0 \%$ & $6.7 \%$ & $6.7 \%$ & $100.0 \%$ \\
\hline 15. & I worry about meeting people for the first time & $\mathrm{n}$ & 2 & 9 & 2 & 0 & 2 & 15 \\
\hline & & $\%$ & $13.3 \%$ & $60.0 \%$ & $13.3 \%$ & $0.0 \%$ & $13.3 \%$ & $100.0 \%$ \\
\hline 16. & I lack confidence when I am out socially & $\mathrm{n}$ & 2 & 5 & 6 & 0 & 2 & 15 \\
\hline & & $\%$ & $13.3 \%$ & $33.3 \%$ & $40.0 \%$ & $0.0 \%$ & $13.3 \%$ & $100.0 \%$ \\
\hline 17. & I often stare at other people's teeth & $\mathrm{n}$ & 2 & 5 & 5 & 3 & 0 & 15 \\
\hline & & $\%$ & $13.3 \%$ & $33.3 \%$ & $33.3 \%$ & $20.0 \%$ & $0.0 \%$ & $100.0 \%$ \\
\hline 18. & I often stare at other people's face & $\mathrm{n}$ & 2 & 2 & 6 & 4 & l & 15 \\
\hline & & $\%$ & $13.3 \%$ & $13.3 \%$ & $40.0 \%$ & $26.7 \%$ & $6.7 \%$ & $100.0 \%$ \\
\hline 19. & I worry that people will make hurtful comments about my & $\mathrm{n}$ & 2 & 6 & 5 & 0 & 2 & 15 \\
\hline & facial appearance & $\%$ & $13.3 \%$ & $40.0 \%$ & $33.3 \%$ & $0.0 \%$ & $13.3 \%$ & $100.0 \%$ \\
\hline 20. & I sometimes get depressed about my appearance & $\mathrm{n}$ & 2 & 5 & 7 & 0 & 1 & 15 \\
\hline & & $\%$ & $13.3 \%$ & $33.3 \%$ & $46.7 \%$ & $0.0 \%$ & $6.7 \%$ & $100.0 \%$ \\
\hline 21. & I sometimes think that people are staring at me & $\mathrm{n}$ & 2 & 5 & 5 & 1 & 2 & 15 \\
\hline & & $\%$ & $13.3 \%$ & $33.3 \%$ & $33.3 \%$ & $6.7 \%$ & $13.3 \%$ & $100.0 \%$ \\
\hline 22. & comments about my appearance really upset me, & $\mathrm{n}$ & 1 & 8 & 4 & 0 & 2 & 15 \\
\hline & although I know they are just joking & $\%$ & $6.7 \%$ & $53.3 \%$ & $26.7 \%$ & $0.0 \%$ & $13.3 \%$ & $100.0 \%$ \\
\hline
\end{tabular}

Table 3: Patient response 6 months after the completion of orthodontic and orthognathic surgery (T2)

\begin{tabular}{|c|c|c|c|c|c|c|c|c|}
\hline \multirow{2}{*}{$\begin{array}{l}\text { Sl } \\
\text { No. }\end{array}$} & \multirow[t]{2}{*}{ OQLQ } & \multirow{2}{*}{$\begin{array}{l}\mathbf{N} \\
\% \\
\end{array}$} & \multicolumn{5}{|c|}{ Scores } & \multirow[t]{2}{*}{ Total } \\
\hline & & & 0 & 1 & 2 & 3 & 4 & \\
\hline \multirow[t]{2}{*}{1.} & \multirow{2}{*}{$\begin{array}{l}\text { I am self-conscious about the appearance of my } \\
\text { teeth }\end{array}$} & $\mathrm{n}$ & 0 & 0 & 2 & 8 & 5 & 15 \\
\hline & & $\%$ & $0.0 \%$ & $0.0 \%$ & $13.3 \%$ & $53.3 \%$ & $33.3 \%$ & $100.0 \%$ \\
\hline \multirow[t]{2}{*}{2.} & \multirow[t]{2}{*}{ I don't like seeing a side view of my face (profile) } & $\mathrm{n}$ & 15 & 0 & 0 & 0 & 0 & 15 \\
\hline & & $\%$ & $100.0 \%$ & $0.0 \%$ & $0.0 \%$ & $0.0 \%$ & $0.0 \%$ & $100.0 \%$ \\
\hline \multirow[t]{2}{*}{3.} & \multirow{2}{*}{$\begin{array}{l}\text { I spend a lot of time studying my face in the } \\
\text { mirror }\end{array}$} & $\mathrm{n}$ & 2 & 0 & 6 & 5 & 2 & 15 \\
\hline & & $\%$ & $13.3 \%$ & $0.0 \%$ & $40.0 \%$ & $33.3 \%$ & $13.3 \%$ & $100.0 \%$ \\
\hline \multirow[t]{2}{*}{4.} & \multirow{2}{*}{$\begin{array}{l}\text { I spend a lot of time studying my teeth in the } \\
\text { mirror }\end{array}$} & $\mathrm{n}$ & 2 & 2 & 5 & 4 & 2 & 15 \\
\hline & & $\%$ & $13.3 \%$ & $13.3 \%$ & $33.3 \%$ & $26.7 \%$ & $13.3 \%$ & $100.0 \%$ \\
\hline \multirow[t]{2}{*}{5.} & \multirow[t]{2}{*}{ I dislike having my photograph taken } & $\mathrm{n}$ & 10 & 5 & 0 & 0 & 0 & 15 \\
\hline & & $\%$ & $66.7 \%$ & $33.3 \%$ & $0.0 \%$ & $0.0 \%$ & $0.0 \%$ & $100.0 \%$ \\
\hline \multirow[t]{2}{*}{6.} & \multirow[t]{2}{*}{ I dislike being seen on video } & $\mathrm{n}$ & 11 & 4 & 0 & 0 & 0 & 15 \\
\hline & & $\%$ & $73.3 \%$ & $26.7 \%$ & $0.0 \%$ & $0.0 \%$ & $0.0 \%$ & $100.0 \%$ \\
\hline \multirow[t]{2}{*}{7.} & \multirow[t]{2}{*}{ I am self conscious about my facial appearance } & $\mathrm{n}$ & 2 & 2 & 6 & 3 & 2 & 15 \\
\hline & & $\%$ & $13.3 \%$ & $13.3 \%$ & $40.0 \%$ & $20.0 \%$ & $13.3 \%$ & $100.0 \%$ \\
\hline
\end{tabular}


\begin{tabular}{|l|l|}
\hline 8. & I do not like smiling when I am meeting people
\end{tabular}

9. I have problems biting

10. I have problems chewing

11. There are some foods I avoid eating because the way my teeth meet make them difficult

12. I get pain in my face or jaw

13. I don't like eating in public places

14. I try to cover my mouth when I meet people for the first time

15. I worry about meeting people for the first time

16. I lack confidence when I am out socially

17. I often stare at other people's teeth

18. I often stare at other people's face

19. I worry that people will make hurtful comments about my facial appearance

20. I sometimes get depressed about my ppearance

21. I sometimes think that people are staring at me

22. comments about my appearance really upset me, although I know they are just joking

\begin{tabular}{|c|c|c|c|c|c|c|}
\hline $\mathrm{n}$ & 13 & 1 & 0 & 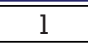 & 0 & 15 \\
\hline$\%$ & $86.7 \%$ & $6.7 \%$ & $0.0 \%$ & $6.7 \%$ & $0.0 \%$ & $100.0 \%$ \\
\hline $\mathrm{n}$ & 9 & 3 & 2 & 0 & l & 15 \\
\hline$\%$ & $60.0 \%$ & $20.0 \%$ & $13.3 \%$ & $0.0 \%$ & $6.7 \%$ & $100.0 \%$ \\
\hline $\mathrm{n}$ & 11 & 4 & 0 & 0 & 0 & 15 \\
\hline$\%$ & $73.3 \%$ & $26.7 \%$ & $0.0 \%$ & $0.0 \%$ & $0.0 \%$ & $100.0 \%$ \\
\hline $\mathrm{n}$ & 11 & 4 & 0 & 0 & 0 & 15 \\
\hline$\%$ & $73.3 \%$ & $26.7 \%$ & $0.0 \%$ & $0.0 \%$ & $0.0 \%$ & $100.0 \%$ \\
\hline $\mathrm{n}$ & 11 & 4 & 0 & 0 & 0 & 15 \\
\hline$\%$ & $73.3 \%$ & $26.7 \%$ & $0.0 \%$ & $0.0 \%$ & $0.0 \%$ & $100.0 \%$ \\
\hline $\mathrm{n}$ & 9 & 6 & 0 & 0 & 0 & 15 \\
\hline$\%$ & $60.0 \%$ & $40.0 \%$ & $0.0 \%$ & $0.0 \%$ & $0.0 \%$ & $100.0 \%$ \\
\hline $\mathrm{n}$ & 12 & 3 & 0 & 0 & 0 & 15 \\
\hline$\%$ & $80.0 \%$ & $20.0 \%$ & $0.0 \%$ & $0.0 \%$ & $0.0 \%$ & $100.0 \%$ \\
\hline $\mathrm{n}$ & 15 & 0 & 0 & 0 & 0 & 15 \\
\hline$\%$ & $100.0 \%$ & $0.0 \%$ & $0.0 \%$ & $0.0 \%$ & $0.0 \%$ & $100.0 \%$ \\
\hline $\mathrm{n}$ & 11 & 4 & 0 & 0 & 0 & 15 \\
\hline$\%$ & $73.3 \%$ & $26.7 \%$ & $0.0 \%$ & $0.0 \%$ & $0.0 \%$ & $100.0 \%$ \\
\hline $\mathrm{n}$ & 3 & 5 & 5 & 2 & 0 & 15 \\
\hline$\%$ & $20.0 \%$ & $33.3 \%$ & $33.3 \%$ & $13.3 \%$ & $0.0 \%$ & $100.0 \%$ \\
\hline $\mathrm{n}$ & 3 & 2 & 6 & 3 & 1 & 15 \\
\hline$\%$ & $20.0 \%$ & $13.3 \%$ & $40.0 \%$ & $20.0 \%$ & $6.7 \%$ & $100.0 \%$ \\
\hline $\mathrm{n}$ & 14 & 1 & 0 & 0 & 0 & 15 \\
\hline$\%$ & $93.3 \%$ & $6.7 \%$ & $0.0 \%$ & $0.0 \%$ & $0.0 \%$ & $100.0 \%$ \\
\hline $\mathrm{n}$ & 11 & 4 & 0 & 0 & 0 & 15 \\
\hline$\%$ & $73.3 \%$ & $26.7 \%$ & $0.0 \%$ & $0.0 \%$ & $0.0 \%$ & $100.0 \%$ \\
\hline $\mathrm{n}$ & 3 & 3 & 5 & 4 & 0 & 15 \\
\hline$\%$ & $20.0 \%$ & $20.0 \%$ & $33.3 \%$ & $26.7 \%$ & $0.0 \%$ & $100.0 \%$ \\
\hline $\mathrm{n}$ & 10 & 5 & 0 & 0 & 0 & 15 \\
\hline$\%$ & $66.7 \%$ & $33.3 \%$ & $0.0 \%$ & $0.0 \%$ & $0.0 \%$ & $100.0 \%$ \\
\hline
\end{tabular}

Table 4: Comparison of esthetic outcome between timelines T0,Tl and T2

Esthetic component

\begin{tabular}{|l|l|l|l|l|l|l|} 
T0 (SD) & T1 (SD) & T value & P value (T0-T1) & T2 (SD) & T value & P value (T0-T2) \\
\hline
\end{tabular}

\begin{tabular}{|l|l|l|l|l|}
\hline I am self-conscious about the appearance & 2.60 (1.24) & $3.13(0.99)$ & 1.30 & 0.20
\end{tabular}

of my teeth

\begin{tabular}{|l|l|l|l|l|}
\hline I don't like seeing a side view of my face & 2.93 (1.09) & 2.93 (1.09) & 0.0
\end{tabular}

(profile)

\begin{tabular}{|l|l|l|l|l|l}
\hline I spend a lot of time studying my face in the 2.66 (1.11) & 2.80 (1.14) & 0.32
\end{tabular}

mirror

I spend a lot of time studying my teeth in the 2.60 (1.18)

mirror

I dislike having my photograph taken

I dislike being seen on video

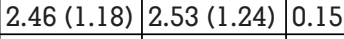

$2.26(1.23) \quad 2.33(1.29) \quad 0.14$

1.0

1.0
0.7

0.74

0.88

0.88

0.87

I am self-conscious about my facial

appearance

I do not like smiling when I am meeting

people

\begin{tabular}{|l|l|l|l|l}
\hline $2.53(1.06)$ & $2.66(1.11)$ & 0.33 & 0.74
\end{tabular}

\begin{tabular}{l|l|l|l|}
$2.40(0.91)$ & $2.13(1.12)$ & 0.71 & 0.48
\end{tabular}

\begin{tabular}{|l|l|l|}
\hline $3.20(0.67)$ & 1.64 & 0.11 \\
\hline 0 & 10.3 & $0.00^{* *}$ \\
\hline $2.33(1.17)$ & 0.79 & 0.43 \\
\hline $2.13(1.24)$ & 1.05 & 0.30 \\
\hline $0.33(0.48)$ & 6.43 & $0.00^{* *}$ \\
\hline $0.26(0.45)$ & 5.93 & $0.00^{* *}$ \\
\hline $2.06(1.22)$ & 1.11 & 0.27 \\
\hline $0.26(0.79)$ & 6.82 & $0.00^{* *}$ \\
\hline
\end{tabular}

The outcome between the time lines T0 and T1 , T0 and T2 were compared. $\mathrm{P}<0.05$ was considered statistically significant.

Table 5: Comparison of functional outcome between timelines T0, T1 and T2

\begin{tabular}{|l|l|l|l|l|l|l|l|}
\hline Functional components & T0 & T1 & T value & Pvalue (T0-T $)$ & T2 & T value & P value (T0-T2) \\
\hline I have problems biting & $1.80(1.08)$ & $1.26(1.22)$ & 1.26 & 0.21 & $0.73(1.16)$ & 2.60 & $0.01^{*}$ \\
\hline I have problems chewing & $2.13(1.24)$ & $1.26(0.96)$ & 2.13 & $0.04^{*}$ & $0.26(0.45)$ & 5.44 & $0.00^{* *}$ \\
\hline $\begin{array}{l}\text { There are some foods I avoid eating } \\
\text { because the way my teeth meet make them } \\
\text { difficult }\end{array}$ & $1.33(0.97)$ & $0.60(1.05)$ & 1.97 & 0.06 & $0.26(0.45)$ & 3.83 & $0.001^{*}$ \\
\hline I get pains in my face or jaw & $1.06(1.09)$ & $1.60(1.05)$ & 1.35 & 0.18 & $0.26(0.45)$ & 2.61 & $0.01^{*}$ \\
\hline
\end{tabular}

The outcome between the time lines T0 and T1 , T0 and T2 were compared. $\mathrm{P}<0.05$ was considered statistically significant.

Table 6: Comparison of social outcome between timelines T0, T1 and T2

\begin{tabular}{|l|l|l|l|l|l|l|l|}
\hline Social components & T0 & Tl & T value & P value (T0-T1) & T2 & T value & P value (T0-T2) \\
\hline I don't like eating in public places & $1.66(0.81)$ & $2.46(1.35)$ & 1.95 & 0.06 & $0.40(0.51)$ & 5.10 & $0.00^{* *}$ \\
\hline $\begin{array}{l}\text { I try to cover my mouth when I meet } \\
\text { people for the first time }\end{array}$ & $1.46(1.06)$ & $1.53(1.12)$ & 0.17 & 0.86 & $0.20(0.41)$ & 4.31 & $0.00^{* *}$ \\
\hline $\begin{array}{l}\text { I worry about meeting people for the first } \\
\text { time }\end{array}$ & $1.33(0.97)$ & $1.40(1.18)$ & 0.18 & 0.86 & 0 & 5.29 & $0.00^{* *}$ \\
\hline I lack confidence when I am out socially & $2.20(1.08)$ & $1.66(1.17)$ & 1.29 & 0.21 & $0.26(0.45)$ & 6.37 & $0.00^{* *}$ \\
\hline
\end{tabular}

The outcome between the time lines T0 and T1 , T0 and T2 were compared. $\mathrm{P}<0.05$ was considered statistically significant. 
Table 7: Comparison Of Psychological Outcome Between Timelines T0, T1 And T2

\begin{tabular}{|l|l|l|l|l|l|l|l|}
\hline Psychological components & T0 & Tl & T value & P value (T0-T1) & T2 & T value & P value (T0-T2) \\
\hline I often stare at other people's teeth & $1.46(0.91)$ & $1.60(0.98)$ & 0.38 & 0.70 & $1.40(0.98)$ & 0.19 & 0.85 \\
\hline $\begin{array}{l}\text { I often stare at other people's face } \\
\text { I worry that people will make hurtful } \\
\text { comments about my facial appearance }\end{array}$ & $1.86(1.12)$ & $2.0(1.13)$ & 0.32 & 0.75 & $1.80(1.20)$ & 0.15 & 0.87 \\
\hline $\begin{array}{l}\text { I sometimes get depressed about my } \\
\text { appearance }\end{array}$ & $1.86(0.99)$ & $1.53(0.99)$ & 0.92 & 0.36 & $0.06(0.25)$ & 5.50 & $0.00^{* *}$ \\
\hline $\begin{array}{l}\text { I sometimes think that people are staring } \\
\text { at me }\end{array}$ & $2.13(1.12)$ & $1.73(1.22)$ & 0.93 & 0.36 & $0.26(0.45)$ & 5.67 & $0.00^{* *}$ \\
\hline $\begin{array}{l}\text { comments about my appearance really } \\
\text { upset me, although I know they are just } \\
\text { joking }\end{array}$ & $2.26(0.96)$ & $1.60(1.12)$ & 1.74 & 0.09 & $0.33(0.48)$ & 6.94 & $0.00^{* *}$ \\
\hline
\end{tabular}

Table 4 to 7 shows the comparison of esthetic, functional, social and psychological outcomes between the timelinesT0 and $\mathrm{Tl}$ and T2. From the table it is evident that as the treatment progressed changes were noted in the patient's attitude and quality of life. Few parameters were found to be statistically significant indicating that the treatment outcome did create $a$ positive change in the patients by correcting their skeletal and dental abnormalities.

\section{DISCUSSION}

A total of 15 patients with dental-skeletal discrepancies participated in the study. Out of them 9 were females and 6 were males. All patients completed the survey within the three time lines. The questions were divided into 4 categories: esthetic, functional, social and psychological. The first 8 questions were based on the esthetic concern of the patient. At time line T0 most of the patients gave scores ranging from 2 to 4 indicating that they were worried about their looks. Even during the timeline $\mathrm{Tl}$ when they were undergoing orthodontic treatment, the response recorded was in the same range. This may be partly because the added presence of fixed braces in the mouth may be a cause for concern; and also because of a phenomenon known as "reverse orthodontics" observed in patients with skeletal -dental combined abnormailites. The patients' when asked said they liked checking the changes in the appearance every time they consulted the Orthodontist during the monthly visits. The responses 6 months after the completion of the whole treatment (T2) mostly ranged from 2-4 suggesting that patients now being aware that their facial and dental features have been modified spend more time watching themselves in the mirror. The"don'tlike"factors have now changed to "like" factors. The happiness quotient was also reflected in their overall attitude and personality.

The problems with biting, chewing, avoiding certain food stuffs and pain in the jaws were fixed as the treatment progressed. All the parameters when compared were statistically significant suggesting that the correction of dental - skeletal malocclusion in turn has positive influence on the dental occlusion its and the harmonious balance with the structures within the oral cavity and surrounding the oral cavity. The results were comparable to previous studies conducted which evaluated the functional wellbeing of the patients before and after combined therapy. ${ }^{9-11}$

The social and psychological factors checked in this study could be seen as an extension of the esthetic component of the questionnaire. The social settings prevailing in this part of the society can be noted in the response to the questions enquiring about the social and psychological health of the patients. The social gatherings here are considered as a platform to meet potential bride or groom candidate for one's offspring. Most of the patients in our study were in the age range of 18-25 years. This is the time when parents seek marriage alliance for their children. Staring at other peoples' face and teeth is a very common finding in this region. So naturally people who are worried about their esthetic features refrain themselves from social gatherings and are often tagged as being "shy". This can be observed in the results of the study (Table 5\&6). Aesthetically pleasing face irrespective of the colour of the skin is a much sought after feature. Esthetics has very high impact on the social and mental health of an individual in this region. The patient assumes that he or she is being watched, and that his/her appearance will become a matter of discussion among the members of the society. But at the end of the combined treatment, the results in our study show that there is statistically significant change in the social and psychological make-up of the person. The make over obtained as a result of the treatment has helped metamorphosis of our patients from shy to smart and selfconfident individuals. The results are comparable to other studies that have examined these aspects of patient characteristics. ${ }^{13-16}$ From the results of the present study, it can be summarized that the esthetic, functional, social and psychological outcomes of patients who have undergone combined orthodontic and orthognathic treatments are better post operatively.

\section{REFERENCES}

1. Bailey LJ,Proffit WR,White R Jr:Assessment of patients for orthognathic surgery. Sem in Orthod 1999; 5:209-222.

2. Ostler S, Kiyak A: Treatment expectations versus outcomes among orthognathic surgery patients. Int J Adult Orthodon Orthognath Surg 1991; 6:247-255.

3. Olson RE, Laskin DM:Expectations of patients from orthognathic surgery. J Oral Surg 1980; 38:283-285.

4. Lateefa AlKharafi, Dalal AlHajery, Lars Andersson. Orthognathic Surgery: Pretreatment Information and Patient Satisfaction. Med Princ Pract 2014;23:218-224

5. Faezeh Eslamipour, Arash Najimi, Azade Tadayonfard, Zeinab Azamian. Impact of Orthognathic Surgery on Quality of Life in Patients withDentofacial Deformities. Int J Dent2017, Article ID 4103905, 6pages.

6. Alves e Silva AC, Carvalho RAS, Santos TS, Rocha NS, Gomes ACA, Oliveira e Silva ED. Evaluation of life quality of patients submitted to orthognathic surgery. Dental Press J Orthod.2013;18(5):107-114.

7. S. J. Cunningham, A.M. Garratt, and N. P.Hunt, "Development of a conditionspecific quality of life measure for patients with dentofacial deformity: II. Validity and responsiveness testing," Com Dent Oral Epidem 2002,30(2):81-90.

8. S. J. Cunningham, A.M. Garratt, and N. P.Hunt, "Development of a condition specific quality of life measure for patients with dentofacial deformity: I. Reliability of the instrument, "Com Dent Oral Epidem 2000:28(3):195-201.

9. S. Lee, C.McGrath, and N. Samman, "Quality of life in patients with dentofacial deformity: a comparison of measurement approaches," Int J Oral Maxillofac Surg2007;36(6):488-492.

10. W. S. Choi, S. Lee, C. McGrath, and N. Samman, "Change in quality of life after combined orthodontic-surgical treatment of dentofacial deformities, Oral Surg Oral Med Oral Pathol Oral Radiol Endodont2010;109(1):46-51.

11. E. Motegi, J. P. Hatch, J. D. Rugh, and H. Yamaguchi, "Health related quality of life and psychosocial function 5 years after orthognathic surgery," Am J Orthod Dentfac Orthoped 2003;124(2):138-143.

12. R. J. Fonseca, R. D. Marciani, T. A. Turvey, and J. Scully, Oral and Maxillofacial Surgery,2009.

13. M. Modig, L. Andersson, and I.W ${ }^{\circ}$ ardh, "Patients' perception of improvement after orthognathic surgery: Pilot study," Br J Oral Maxillofac Surg2006;44(1):24-27.

14. E. Desforges, R. Mathis, A. Wilk et al. "The psychological impact of orthognathic surgery," L' Orthodontie Franc aise2007:78(2):113-121.

15. O. T. Hunt, C. D. Johnston, P. G. Hepper, and D. J. Burden,"The psychosocial impact of orthognathic surgery:A systematic review," Am J Orthod DentfacOrthoped2001;120(5):490-496.

16. R. H. Pahkala and J. K. Kellokoski, "Surgical-orthodontic treatment and patients' functional and psychosocial well-being," Am J Orthod Dent fac Orthoped2007;132(2):158-164. 